\title{
Dynamic Netvalue Analyzer - A Pricing Plan Modeling Tool for ISPs Using Actual Network Usage Data
}

\author{
Jörn Altmann \\ Internet and Mobile Systems Department \\ CMSL, Hewlett-Packard Labs \\ jorn_altmann@hpl.hp.com
}

\author{
Lee Rhodes \\ Usage Management Division \\ OpenView Organization, Hewlett-Packard Company \\ lee_rhodes@hp.com
}

\begin{abstract}
The current situation in the Internet service provider market shows that Internet service providers (ISPs) are still struggling to find sustainable business models. The challenge for them is to find the right prices and pricing plans for their network services. In order to satisfy business objectives, prices and pricing plans have to be designed such that they are attractive to end-users but also guarantee revenues. In order to achieve that, it is necessary to have a precise understanding of the usage behavior of end-users under different pricing plans. In this paper, we present existing pricing plans in the market, and discus their advantages and shortcomings. As the result of our analysis, we propose a new pricing plan, which is attractive to end-users as well as ISPs. However, in order to smooth the migration of end-users from existing pricing plans to the new plan, marketing and business managers need support. This could be done with the help of a business intelligence tool, which can provide them with detailed information about user behavior. Such a tool for supporting service providers in pricing network services is presented in this paper. This tool, which is called HP OpenView Dynamic Netvalue Analyzer, helps to analyze and model pricing plans and prices. We demonstrate capability of DNA by describing the creation of a tiered, usage-based pricing plan based on real network usage data under a flat-rate pricing plan.
\end{abstract}

\section{Introduction}

We envision an Internet environment that enables endusers to be permanently connected. In such a setting, today's pricing plans such as connect-time-based pricing plans or pure flat-rate pricing plans would not be successful. Connect-time-based pricing plans, where the charges are based on the time the end-user is connected to the Internet, would over-charge end-users since it does not reflect the actual usage of the network. Flat-rate pricing plans, where the end-user is charged a monthly fee for unlimited access, have been shown to be economically inefficient. Studies have shown that $20 \%$ of all end-users generating $80 \%$ of the usage [3]. In order to prevent this subsidy of heavy users, data-volume-based pricing plans are a more appropriate mean to charge for Internet usage.

However, the Internet service provider market seems to be reluctant to adopt these mechanisms, despite the proliferation of broadband access to the Internet and continuous growth of demand for network services. The demand will no doubt grow at a mounting rate, not only because of a continuously increasing customer base, but also because of new emerging Internet applications making use of the available, high bandwidth. In the worse case, the demand grows faster than service providers can bring on extra capacity in metropolitan areas.

The reason that Internet service providers (ISPs) are reluctant is twofold. On the one hand, ISPs would have to invest into a new charging and accounting system, which can provide real-time charging advice to the end-user as well as to account for data gathered from many geographically disperse sources. On the other hand, Internet service providers do not have the tools necessary to estimate and predict the change in their revenue streams, if they introduce usage-based pricing plans into their pricing structures. A financial business analysis tool could help service providers not only to understand these changes but also rapidly customize services and, therefore, increase customer satisfaction.

Traditional business analysis systems depend on usage records being stored in a database. However, the shear amount of data streams and need for real-time correlation generate requirements for costly storage infrastructure. As these storage systems grow large, timely processing of these records becomes also challenging.

Therefore, new approaches are needed in order to extract key business information from usage data (e.g., financial value and user behavior) without having to store all the usage data first.

In this paper, we describe a technology that enables the creation of statistical models of user behavior directly from actual subscriber usage data. The models are then used for generating financial business information. This kind of extraction of business information is distinctly different from existing systems, where there are weak linkages between usage data and marketing information. 
The paper proceeds as follows: In the next section we give an overview of pricing plans in the network service provider market and discuss the requirements and prerequisites of potential new pricing plans. The third section introduces the proposed pricing plan. Dynamic Netvalue Analyzer (DNA), a business analysis and pricing plan modeling tool for network service providers, is presented in section 4. How DNA helps to transform flatrate pricing plans into tiered, usage-based pricing plans is shown in section 5 . We close the paper with a short discussion of our experiments with DNA in section 6 .

\section{Current pricing plans in the ISP market}

Although many kinds of different pricing plans could be conjured, only a few are actually deployed in the network service provider market. The two dominant kinds of pricing plans are connect-time-based pricing plans and flat-rate pricing plans. While flat-rate pricing plans are dominant in the USA market, Europe and Asia offer connect-time-based pricing plans. However, both pricing plans restrain further market growth in the broadband access market as well as in the $3 \mathrm{G}$ wireless access market.

\subsection{Connect-time-based pricing plans}

Under the connect-time-based pricing plan, which is a pure usage-based pricing plan (Figure 1), the end-user pays for the time he is connected to the Internet (i.e. usage). If he did not connect to the Internet at all for a month, he would owe nothing. Since this usage-based pricing plan is independent to the actual amount of transmitted data, this pricing model does not reflect the actual cost (or utility) of using a packet-based network. However, it is a very prevalent type of plan. Light endusers, who are only using the Internet to send and receive emails, appreciate this pricing plan, since it is less expensive than a flat-rate pricing plan.

The main reason for the implementation of connecttime-based pricing plans is that ISPs can utilize the billing and charging system of the PSTN. In this case, no new investment in a system for charging and billing is required.

However, this kind of pricing can inhibit light end-users from extensively exploring new Internet media. Light users might evaluate the cost (risk) for the exploration of the Internet to be higher than the utility that they expect to receive from its exploration. Therefore, connect-timebased pricing plans curb revenues based on usage growth.

\subsection{Flat-rate pricing plans}

Under the flat-rate plan, a user pays a fixed amount B per a fixed amount of time for unlimited usage (Figure 1). Flat-rate pricing plans do not have the shortcoming of discouraging the exploration of new services on the Internet. It is also attractive to end-users because of the predictability of charges and the fact that they are not bothered with a ticking usage meter (Odlyzko [5]).

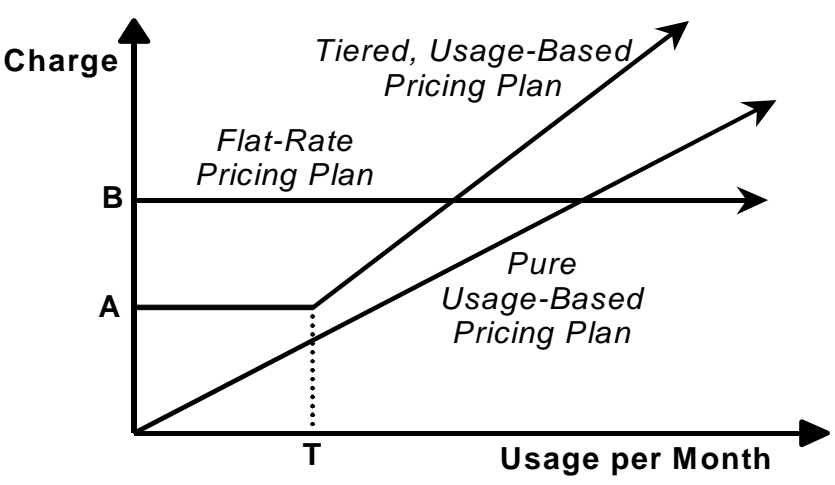

Figure 1. Comparison of Pricing Plans

Once end-users decided to pay the flat fee at the beginning of each month, they face only time cost for using the Internet. From the service provider perspective, the advantages of flat-rate pricing plans are that they are easy to bill and that they provide a predictable annuity.

However, flat-rate pricing brings its own set of shortcomings. The shortcomings are the lack of fairness, the deterioration of service quality through over-usage, as well as the restraint of further market growth in the broadband access market.

Because of the heterogeneity of the end-user population and the different kind of applications, the amount of data transmitted within a fixed time period varies over an enormous range. Altmann, Rupp, and Varaiya give an example, in which the monthly usage varies across 6 orders of magnitude [3]. With broadband access, the variation is even greater - as much as 8 to 10 orders of magnitude (Figure 5). Additionally, heavy users overwhelmingly dominate the consumption of bandwidth. Results show that 20 percent of users on a network consume 75 percent of the resource. Concluding these facts, it means that not only the light end-users, but also the middle spectrum of users are effectively subsidizing the heavy users under a monthly fixed, flat-rate pricing plan. The end result is a strong lack of fairness.

Another shortcoming of flat-rated pricing is that network congestion is inevitable, resulting in service quality deterioration. Once the flat rate is paid, there is no monetary limit to the usage of the network. For example, end-users with a low valuation of their time (e.g. teenagers) will use the Internet extensively. There is no incentive to reduce usage if the network gets congested. Additionally, there is no incentive to manage usage. The consequence is network congestion that reduces the quality of service for everyone. Therefore, the need of customers desiring Internet access quality cannot be satisfied. This is 
especially true for broadband access customers, since they are concerned about access quality (they chose to pay a higher fee than for dial-up service). Consequently, a large proportion of these customers become unsatisfied.

In a scenario where the demand continuously increases and service providers charge a flat rate, the only recourse for service providers is to increase the fixed fee for everyone. This is a poor solution since the increased prices will not only force lower income customers to quit the service, but also the most lucrative customers (the light and middle-spectrum users) to quit the service. They will look for a service with a better price/quality ratio. Only heavy users will stay with the service. In addition, potential customers may be priced out of the service and be unable to participate, if the fixed price broadband services become just too expensive. This creates a negative reinforcing feedback cycle that will eventually make the service unprofitable. This is a classic lose-lose financial scenario for service provider. In general, it restrains further market growth.

\section{The proposed pricing plan}

Looking at the current situation in the Internet service provider market, the question poses what kind of pricing plan can increase customer satisfaction as well as proliferate further growth and return on investment (ROI) in the broadband and $3 \mathrm{G}$ wireless Internet market.

\subsection{Pricing plan requirements}

Considering the pros and cons of connect-time-based pricing plans and flat-rate pricing plans, we gain some insight about the important characteristics that a new pricing plan should have. The pricing plan should encourage end-users to behave responsibly (i.e. either consuming fewer resources or paying a higher usage fee if there is congestion). This can be achieved by introducing a usage-based component to the pricing plan. In addition to this, the usage-based component helps allocating network resources in a fair way. Light users end up with lower fees and heavy users are forced to reduce their consumption. The resources freed can be used to attract new light users. However, the pricing plan must have a low entry-level fee for light and new users as well. This component of the pricing plan should provide predictable charges, encouraging end-users to stay permanently connected to the Internet without worrying about charges. This will increase both revenues of service providers and customer satisfaction.

The pricing plan should also provide the end-user flexibility as to the quality of service level, since we predict that quality services will always be charged on a usage basis, even in the presence of a flat-rated, best-effort basic service. If users desire high quality network service and are willing to pay for it, they should be able to send their data at high priority. A system, which gives end-users direct access to a price/priority selection at any time, has this capability. It provides variable QoS at fixed price [2].

The frequency of price changes has to be low. We suggest not changing prices more frequently than once every three months.

\subsection{Differentiation of network services}

In the current broadband access market, flat-rate pricing plans are tiered with respect to the peak bandwidth available to the end-user. Using this kind of pricing, ISPs hope to solve the problem of over-usage of resources under flat-rate pricing plans. Although the available bandwidth influences the amount of usage (the higher the available bandwidth, the higher the usage), this pricing method will not work. The reduction of usage is not a result of a more responsible behavior, but caused by limiting the number of different applications used. This occurs as the number of different applications used has a direct relationship to the bandwidth on-hand. By taking this approach, ISPs restrict network usage and, therefore, damage their own business.

Instead, usage-based pricing should be used to discourage over-usage of resources, and peak bandwidth should be used as a network service quality differentiator.

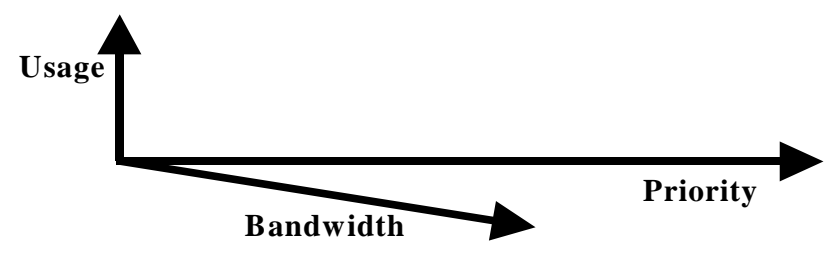

Figure 2. Network service differentiation

The both QoS parameters, which can be experienced by end-users, are bandwidth and network priority (Figure 2). Although both QoS parameters are not independent from each other, they can be used to differentiate the network service. The charge should be based on their actual usage.

\subsection{Pricing plan specification}

The pricing plan that was proposed by Altmann and $\mathrm{Chu}$ is based on bandwidth to differentiate network services [1]. Under this pricing plan, end-users pay a flat rate for a basic service and are charged on a per-usage basis for better-than-best-effort service. However, this pricing plan does not discuss how better-than-best-effort services should be priced and does not consider the broad range of usage difference among end-users.

We propose a modified pricing plan, which considers both issues. The proposed pricing plan is comprised of a 
basic service component and a service flexibility component (Figure 3). Both components together fulfill all requirements of end-users and network service providers.

\section{(2) Service Flexibility Component}

(a) usage-based rate

for consumption of

resources at bandwidth

and priority levels higher

than specified in the Basic

Service Component (b) usage-based rate

for consumption of resources exceeding the data-volume threshold specified in the Basic Service Component

\section{(1) Basic Service Component}

flat rate for consumption of resources up to a datavolume threshold at fixed bandwidth and priority level

\section{Figure 3. The proposed pricing plan}

The basic service component offers network service at a fixed bandwidth and fixed priority level up to a fixed amount of data-volume. Depending on the user's selection of all three values, he pays a certain flat fee for this basic service each month. This component takes care of the enormous difference in the range of usage among endusers. It also delivers predictability of charges for endusers, and predictability of revenues for the network service provider.

The service flexibility component provides the end-user with the ability to select bandwidths and priority levels higher than the one specified in the basic service component. The end-user is required to pay for these services on a pure usage basis. The service flexibility component also specifies the rate that end-users must pay if they exceed their resource allotment as specified in the basic service component. In general, this component provides end-users with the flexibility of selecting the preferred network service, while enabling the network service provider to capture the surplus that end-users are willing to pay for certain tasks and circumstances.

This pricing plan is fair, since it allocates resources to those end-users, who value the service highly. End-users evaluate the data transfer according to the net utility that they expect to receive from the download. If the utility is higher than the incurred cost (i.e. money and time), they will download. Otherwise, they stop the process.

The charge $C$ for such a pricing plan can be calculated using the following formula:

$C=R_{\text {Flat }}\left(B_{b}, P_{b}, U_{b}\right)+\sum_{b, p} x\left(b, p, U_{b}\right) \cdot R_{U s a g e}\left(b, p, B_{b}, P_{b}\right)$,

where $R_{\text {Flat }}$ defines the flat rate for the buy-out of $U_{b}$ bytes at priority $P_{b}$ and bandwidth $B_{b}$. The number of bytes $x$ transmitted at bandwidth $b$ and priority level $p$ above the buy-out threshold is denoted by $x\left(b, p, U_{b}\right)$. $R_{\text {Usage }}$ defines the rate per byte at bandwidth $b$ and priority level $p$, considering the bought-out bandwidth $B_{b}$ and the boughtout priority level $P_{b}$.

\section{Dynamic Netvalue Analyzer}

HP OpenView Dynamic Netvalue Analyzer (DNA) is a business intelligence and decision support tool targeted for network service providers. DNA transforms raw customer usage data into business information, empowering business managers to model revenues and profitability for new and existing services under different pricing plans.

\subsection{Functionality}

DNA helps business managers to understand the usage behavior of their subscribers in real-time. This includes the identification of needs for new services, the task of keeping current subscriber plans price competitive and profitable, the identification of high usage subscribers for potential up-selling or cross-selling of new services, and the identification of low-usage subscribers that may be candidates for churn. In general, it eases customer/service life cycle management, and enables new revenue and return on investment paradigms.

By converting raw usage data into statistical models (streaming data analysis), instead of storing all the raw data as in a warehouse approach, DNA frees storage space, enabling the analysis of more historical data in a shorter time period. The statistical models are based on business dimensions such as pricing plans, services, or geography.

\subsection{Architecture}

DNA is comprised of the backend server, which aggregates the usage data, and clients to view and model pricing plans (Figure 4).

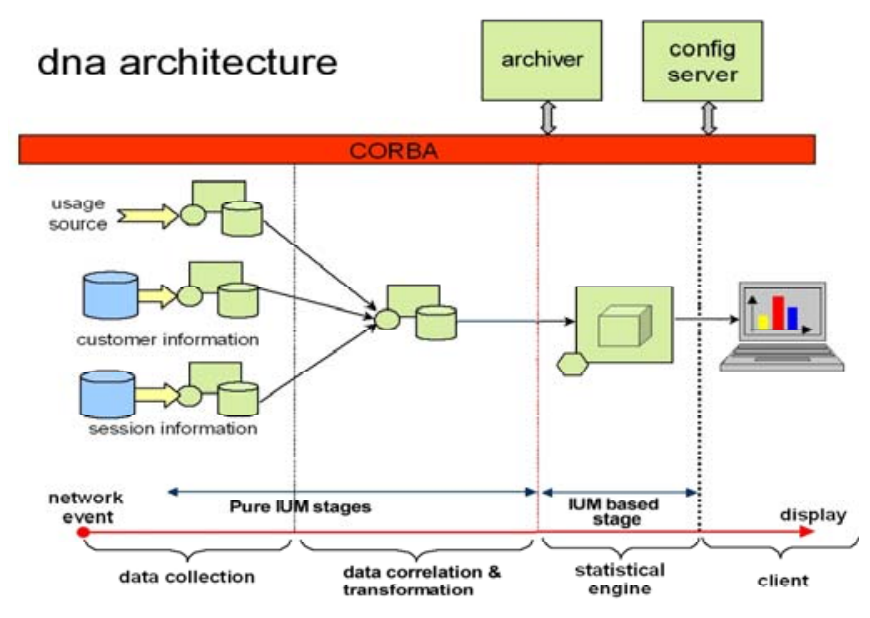

Figure 4. DNA architecture 
The backend server is built on HP's Internet Usage Manager (IUM) mediation platform. At the data collection stage, three modules of IUM are used to aggregate customer information, session information, and the actual usage data of all end-users. The second level correlates the output of the previous stage and transforms the data into an internal format, which allows to rapidly access individual usage data. The third stage is comprised of the statistical models, which are specified by the user of the tool. The models are populated by the second stage's output. The third stage also interacts with clients, which request the model data.

The DNA client consists of two major parts: an analysis tool and a financial modeling tool. Each tool has a separate window, from which usage data can be analyzed and pricing plans can be setup and created.

The analysis tool allows network usage data to be viewed in numerous formats with varying levels of detail (Figure 5). The data is presented in statistical histograms, tables, and summaries. For an in-depth analysis, it allows the business manager to view individual subscriber usage. The usage data can also be compared with historical data.

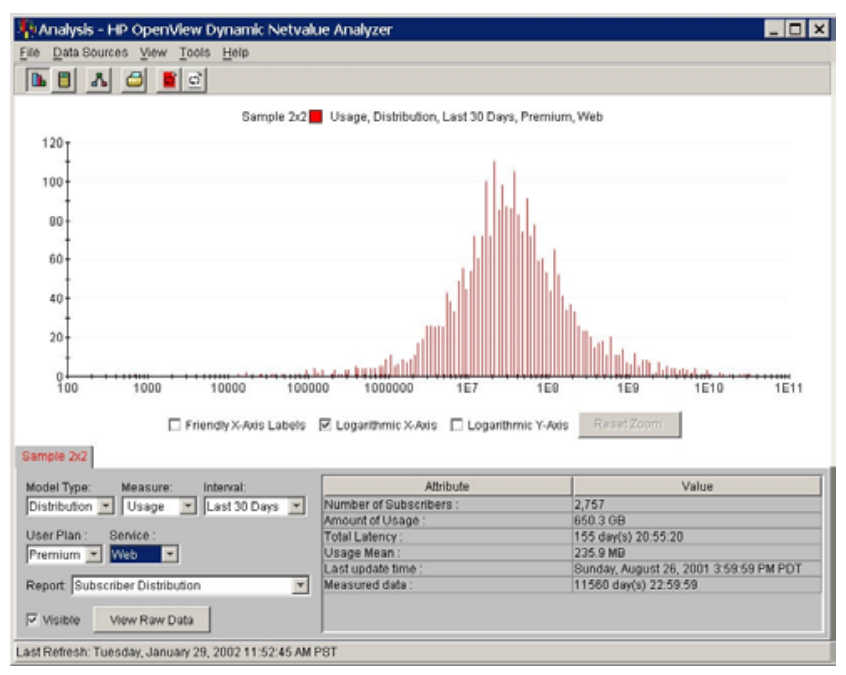

Figure 5. DNA analysis screen

The financial modeling tool is used to develop pricing plans and view modeling results, showing usage, revenue, and profits (Figure 6). It also allows the merging of usage data of customers on different pricing plans.

\section{Modeling of pricing plans: a case study}

The modeling of pricing plans helps smoothing the migration between different pricing plans. It lowers the risk of end-users rejecting new pricing plans.

The migration from connect-time-based pricing plans to data-volume-based pricing plans is simple to achieve, since end-users will easily accept this new way of charging. They are already accustomed to usage-based charges and the new plan clearly provides more benefits.

The migration from pure flat-rate pricing plans to usagebased pricing plans requires more careful planning, as the example of how to migrate from a pure flat-rate pricing plan to a version of the proposed pricing plan will show. We refer to the new plan as the tiered, usage-based pricing plan.

Under this tiered, usage-based pricing plan, the usage measure is assumed to be the total amount of bytes transmitted per month. The end-user buys out a finite, fixed amount of data volume $\mathrm{T}$ for $\mathrm{a}$ fixed fee $\mathrm{A}$ (Figure 1). However, he pays a usage-based charge for the amount of data exceeding the bought-out amount (slope in Figure 1).

Besides setting the right prices, the challenge is to create the right set of tiered, usage-based pricing plans, which replace the flat-rate pricing plan. For determining the number of tiers, several parameters have to be considered, such as the number of users within a tier, the cost for supporting the tier, customer satisfaction, as well as the usage.

The data in this example is real subscriber usage data from one of HP's DNA customers. It represents 2757 users of a cable broadband service with a total usage of about 650 GBytes during a period of 30 days under a flat-rate pricing plan. As shown in Figure 5, the distribution of users with respect to their total usage within 30 days reveals a large variety in usage, ranging from over 8 orders of magnitude. The shape of the graph shows a lognormal distribution.

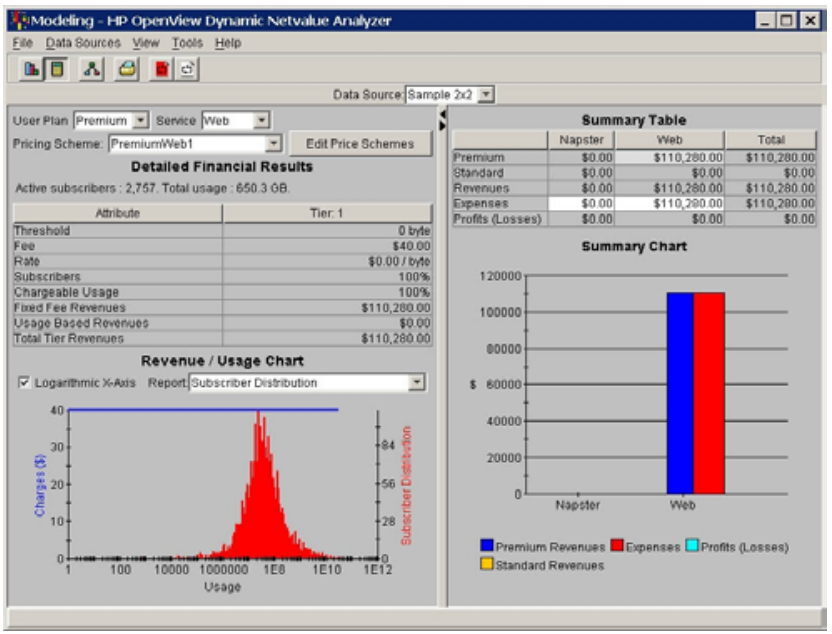

Figure 6. DNA modeling screen showing a flat-rate pricing plan

Assuming that this service provider offers this service at a flat fee of $40 \$ /$ month, the financial modeling tool calculates $\$ 110,280$ in revenues (Figure 6). (Note: In this case study, we are only examining the www service. 
Therefore, the prices of the other services are set to zero.) For the purpose of this case study, the expenses are set equal to the revenues. This helps us in quickly evaluating the modeled pricing plans.

The next step is to determine the usage rate for the user population in order to equal the current revenues. Setting the flat rate to zero, and with some trial-and-error iterations, the usage rate comes to 0.169 \$/MByte.

Keeping the usage-based rate at 0.169 \$/MByte, we introduce a data-volume threshold at 500 MBytes for the flat-rate portion of the pricing plan. In order to get the same revenue as in the pure $\$ 40$ flat rate pricing plan, we explore different flat rates. The result is a flat rate of $15 \$$ /month (Figure 7).

The $40 \$ /$ month flat-rate pricing plan is equivalent to this new plan with a monthly fixed fee of $\$ 15$ and a variable charge of $0.169 \$$ MByte for usage above 500 MByte.

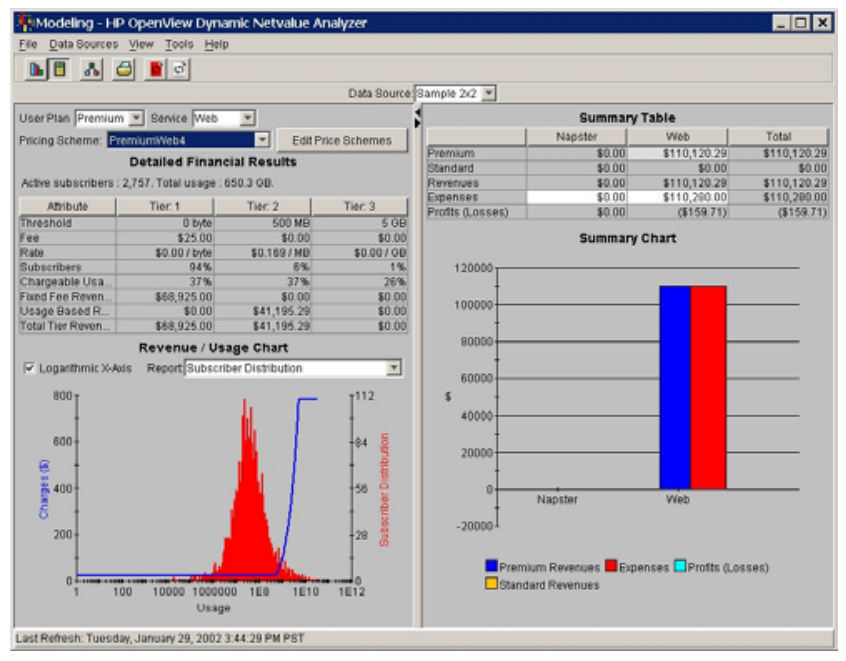

Figure 7. DNA modeling screen showing a tiered, usage-based pricing plan

Advertising this proposed pricing plan, the network service provider could attract many more subscribers than under the $40 \$ /$ month flat-rate pricing plan.

In addition to this, only $6 \%$ of the current customers would be affected by the usage-based component of the pricing plan. This is a low number of customers. Considering the bandwidth consumption of these $6 \%$ of subscribers, the proposed pricing plan is even more justifiable. These $6 \%$ of subscribers are responsible for $76 \%$ of the entire data traffic on the network. They are the heavy users.

However, the calculated fees, which would have to be paid by the heavy users, are unrealistic. They would either change their behavior by using less bandwidth or they would quit the service. In both cases, resources are freed, which could then be sold to more light users, whom are attracted by the lower $\$ 15$ fee.

\section{Conclusion}

Within this paper, we discussed existing pricing plans and pricing principles. After defining requirements for pricing plans and network service quality differentiation, we proposed a new pricing plan, which comprises two components. Under the proposed pricing plan, end-users are charged a basic service fee, which allows them to use the network service at a fixed bandwidth and fixed priority level up to a data-volume threshold. If end-users desire better than this basic service or exceed the threshold, they are charged an additional usage-based fee. This pricing plan meets many requirements of end-users and network service providers with respect to flexibility and simplicity.

However, the migration from existing pricing plans is difficult, since little understanding of user behavior under the new pricing plan exists. The Dynamic Netvalue Analyzer (DNA), a business intelligence tool, eases this problem. It supports ISPs in the migration to usage-based pricing plans, by providing tools to analyze usage behavior of end-users and model pricing plans. The case study presented, the migration to a tiered, usage-based pricing plan, is one of many approaches of how DNA can be used to create pricing plans.

\section{References}

[1] Jörn Altmann and Karyen Chu, "A Proposal for a Flexible Service Plan that is Attractive to Users and Internet Service Provider," IEEE InfoCom2001, Conference on Computer Communication, Ancorage, USA, April 2001.

[2] Jörn Altmann, Hans Daanen, Huw Oliver, and Alfonso Sánchez-Beato Suárez, "How to Market-Manage a QoS Network," IEEE InfoCom2002, Conference on Computer Communications, New York, USA, June 2002.

[3] Jörn Altmann, Björn Rupp, and Pravin Varaiya, "Internet Demand under Different Pricing Schemes," ACM EC1999, Conference on Electronic Commerce (SIGecomm), Denver, Colorado, USA, November 1999.

[4] Anna Bouch and M. Angela Sasse, "Designing QoS and Charging Mechanisms: He Who Pays the Service Shapes the Design," ICQT 2001, Internet Charging and QoS Technology, Vienna, Austria, September 2001.

[5] Andrew M. Odlyzko, "Internet Pricing and the History of Communications," Computer Networks vol.36, 2001.

[6] Dale O. Stahl, Andrew B. Whinston, and Keqiang Zhang, "A Simulation Study of Competitive Internet Pricing: AOL Flat Rates versus GSW Usage Prices," $1^{\text {st }}$ International Conference on Information and Computation Economies, October 1998.

[7] Pravin Varaiya and Richard Edell, "Providing Internet Access: What We Learn From INDEX," IEEE Network, vo13, no5, October 1999. 